The expansion of the geography of mosquito vector regions and their adaptation to colder and drier climatic conditions with the potential for transmission of infection gradually expand the area and require scientific study and monitoring of hemorrhagic fever viruses and Zika virus in particular.

\section{6 doi: 10.15789/2220-7619-2018-4-1.6 MODERN PROBLEMS OF INFECTIOUS DISEASES PREVENTION IN PUBLIC HEALTH OF REPUBLIC OF GUINEA}

O.K. Konstantinov ${ }^{1}$, M.Y. Boiro ${ }^{1}$, N.E. Lama ${ }^{2}$, E.V. Verigina ${ }^{3}$ ${ }^{1}$ Research Institute of Applied Biology of Guinea, Kindia, Republic of Guinea; ${ }^{2}$ Ministry of Health and Public Hygiene, Conakry, Republic of Guinea; ${ }^{3}$ Central Research Institute of Epidemiology, Moscow, Russia

Republic of Guinea is one of the developing countries of West Africa. Of the whole GDP only 400-500 USD are on one habitant annually. More than half habitants live down the poverty level. The country carries a heavy burden of many infectious diseases.

The aim of the study was to describe the actual problems of infectious diseases prevention in public health of Republic of Guinea.

Statistical data from public health institutions of the country were used. Methods - descriptive and analytical

Population size of Guinea increases and numbers now more than 12 millions habitants. The public health structure of the country includes: 925 first-aid posts, 410 prefecture and regional centers of health, 8 communal health centers, 26 prefecture, 7 regional and 3 national hospitals. Though medical aid is below of the regional norms of WHO. In Guinea 74 nosoforms of infectious and non infectious diseases are registered. The part of infectious diseases in the morbidity structure was 38-44\% (1.9-2.5 millions cases annually). The most widespread infections were malaria, acute respiratory (ARI) and intestinal infections. In the mortality structure ARI occupied $12.5 \%$, malaria $-10 \%$, acute intestinal infections $6 \%$, HIV infection consists $5 \%$. The sexual transmissible infections are widespread: 200 thousand cases in a year. The outbreaks of measles, meningitis, cholera, Yellow Jack are registered too. Prevention measures are actively conducted. All little children are vaccinated obligatory against: poliomyelitis (4 ${ }^{\text {th }}$ time), BCG, kombi (diphtheritic, tetanus and pertussis), measles, Yellow Jack. The pregnant women receive anti malaria drugs as prevention and anti tetanus vaccine. The HIV positive pregnant women receive antiretroviral therapy. One realizes health education programs, particulary among the youth. One popularizes the use of contraceptives, organizes centers of family planning. However this activity meets some difficulties because of the low education level, some religious and socio-cultural customs of population, what was visually revealed during Ebola fever outbreak. The vaccination program meets such difficulties as cold chain, lack of qualified medical personnel, lack of medicaments and technical equipment of diagnostics laboratories, which number is insufficient. In order to dissolve these problems of diagnostics, control and prevention of infectious diseases in 2018 the 3 -years program "National strategy on medical biology" was elaborated.

In spite of difficulties the public health of Guinea goes on to develop. The realization of the "National strategy" will help in strengthening of health and welfare of population of Guinea.
1.7

doi: 10.15789/2220-7619-2018-4-1.7

\section{FECAL-ORAL MECHANISM IN THE GROUP AND EPIDEMIC STRUCTURES ON THE TERRITORY OF THE ROSTOV REGION WITHIN 10 YEARS}

E.V. Kovalev, E.G. Erganova, S.A. Nenadskaya, S.S. Slis', G.A. Miroshnichenko

Rostov Region Surveillance Directory, Rostov-on-Don, Russia

Infections with the fecal-oral transmission take one of the leading places in the infection pathology of population including economically developed counties, going only after respiratory infections among mass infectious diseases.

In Rostov region according to the form of statistic observation No. 23-17 "Information about outbreaks of infectious diseases", within 10 years from 2008 until 2017 th 104 cases of group and outbreak morbidity was reported, with 1853 caseload, including 1130 children under 17 years (61.0\% of the total number of cases).

In structure the main gravity in the region lies on the fecal-oral mechanism (76.9\%) and also on aspiration $(10.6 \%)$ and others $(12.5 \%)$.

The spread was occurred by food - in 47 cases ( $45.2 \%)$, by water - in $14(13.5 \%)$, by household contacts - in 19 (18.3\%), airborne-in 11 (10.5\%) and other - 13 (12.5\%)

Etiological factors in group and outbreak morbidity were bacterial pathogens of infection: Salmonella Enteritidis (11), Salmonella Typhimurium (3), Salmonella Seegefeld (1), Salmonella Muenchen (1), Salmonella Isangi (1), Salmonella spp. (1), Shigella sonnei (6), opportunistic pathogenic microflora (7) (Staphylococcus aureus, Proteus vulgaris, Citrobacter, Escherichia coli, Enterobacter aerogenes), viral aetiology: group A rotaviruses (15), 2 genotype Noroviruses (18), hepatitis a virus (9), Enteroviruses (14), mixed etiology (1), undetermined etiology (4), measles virus (5), Crimean-Congo hemorrhagic fever (1), parvovirus in 19 (1), influenza (1), tuberculosis (1), chickenpox (1), community-acquired pneumonia (1) and epidparotite (1).

Improvement of decoding of acute intestinal infections (ОКИ) and further development of laboratory diagnostics in etiological structure dramatically changes were noticed: the increasing epidemiological importance is given to "intestinal" viruses, the intensity of circulation of which has increased in recent years (enteroviruses, rotaviruses and noroviruses).

The changed structure of the epidemic process including acute intestinal infections, the growth of viral infections requires new approaches in the improvement of activities and diagnosis, anti-epidemic supply and prevention activity.

1.8 doi: 10.15789/2220-7619-2018-4-1.8

THE CHARACTER OF MICROBIOTA IN THE INTENSIVE CARE UNIT OF THE CHILDREN'S HOSPITAL AND ITS EPIDEMIOLOGICAL SIGNIFICANCE

L.A. Kraeva ${ }^{1,2}$, L.B. Vainer, K.E. Vinogradova

${ }^{1}$ St. Petersburg Pasteur Institute, St. Petersburg, Russia;

${ }^{2}$ S.M. Kirov Military Medical Academy, St. Petersburg,

Russia; ${ }^{3}$ Pavlov First St. Petersburg State Medical University, St. Petersburg, Russia

As is known, the risk of infections associated with medical care is highest in intensive care units, especially in the department of pathology of newborns and premature infants. The aim of the study was to study the composition and characteristics of bacteria circulating in the intensive care unit of the children's hospital. 
Material for the study: 637 sputum samples from 171 children of the intensive care unit, including from the department of pathology of newborns and premature infants; scrapes from the throat, nose and hands of 53 medical staff; 86 scrapes from the surfaces of the environment. Research methods: bacteriological and MALDI-TOF/MS (Bruker Daltonics).

It was found that 30 species of bacteria were isolated from children, including: Staphylococcus aureus (10\%), Staphylococcus epidermidis (8\%), Klebsiella pneumoniae (7\%), Streptococcus pneumoniae 7\%), Enterococcus faecium (2\%). S. aureus (45\%), S. epidermidis $(90 \%)$, S. pneumoniae $(17 \%)$, K. pneumoniae $(6 \%)$ were isolated from medical personnel serving these children. Staphylococci of different species with the highest proportion of $S$. epidermidis (21\%) were isolated from $50 \%$ of environmental objects. Most often they were isolated in newborns (16\%), less often in children aged 1 month to 1 year $(10 \%)$, in children $1-3$ years $(8 \%)$, in children older than 3 years $(1 \%)$. These data indicate a contact-household route of transmission of infection caused by $S$. epidermidis. The frequent occurrence of $S$. aureus and $S$. pneumoniae in children and medical personnel in the absence of these bacteria in the environment indicates the role of resuscitation department staff as a source of these infections. Bacteria $K$. pneumoniae and E. faecium are isolated from both children, medical personnel and environmental objects. All of the above bacteria were well subjected to destruction by disinfectants. After reorganization activities in the department, the proportion of positive bacterial seeding decreased significantly.

However, the study found that Bacillus cereus strains isolated from newborn infants $(6 \%)$ and from medical and general-purpose equipment are resistant to all disinfectants used in the department. As a result of the research, the drugs to which the isolated bacilli are sensitive were selected.

Thus, continuous monitoring of the microbiota, the study of its characteristics and the epidemiological approach to assessing the situation can significantly reduce or avoid the development of infections associated with the provision of medical care.

\section{9 doi: 10.15789/2220-7619-2018-4-1.9 \\ ETHICS OF VACCINATION AS THE CRITERIA OF THE SCIENTIFIC AND HUMANISTIC APPROACH}

\section{O.I. Kubar}

St. Petersburg Pasteur Institute, St. Petersburg, Russia

The aim of this research was to analyze the unique role of the vaccination from its successful and problematical sides concerning the developing, distribution and using the vaccines in different epidemiological situation in the mirror of the global bioethics. The role of the balance between ethics and success of vaccination was clearly illustrated and based on examples from the history of vaccination and our own experience connected with amazing contribution of Leningrad/St. Petersburg Pasteur Institute in the history of vaccination. It was clear shown that the particular importance has the harmonization and solidarity of all persons and structures involved in the process of vaccine prevention within local, national, regional, and global levels. In order to reach the goal in protection of the infections diseases by vaccination, it is necessary to follow in reality and to demonstrate to the society such universal ethical standards as scientific honesty, social expediency, justice, non-discrimination, transparency and overcoming of the conflict of interests. Detailed description how these ethical principals particularly work in the stage of $R \& D$ vaccines, its distribution, using for vulnerable population and in the frame of WHO program for eradication of poliomyelitis, measles, and rubella was done. The specific relevance of the presented study was to highlight the key role of ethical principles of the adversarial position to the increasing anti-vaccination lobby. It is well known that the success of vaccination is associated not only with effective and safe vaccines, but directly depends on the society adherence to vaccination acceptance and realization. A negative influence of anti-vaccination movement, which promotes mistrust to vaccination, is playing an essential role in this process. The objective analysis and identification of ethical errors in the process of vaccination demonstrated in this research show the way any unethical action is creating a basis for the anti-vaccination movement. The maintaining of ethical standards balance and building partnerships and interaction with society as well as the implementation the ethical elements in legal regulation of the vaccination process on national and international levels are crucial for achieving optimum results in countermeasures against evolving the anti-vaccination lobby and protection against infections by vaccination in the present time and future perspective.

1.10 doi: 10.15789/2220-7619-2018-4-1.10

\section{ETHICAL CONSIDERATION IN CONCEPTION OF INFECTIOUS DISEASES ERADICATION}

O.I. Kubar, M.A. Bichurina, N.I. Romanenkova, N.I. Rosaeva, N.V. Zheleznova, I.N. Lavrentieva, A.Yu. Antipova, O.I. Kanaeva

St. Petersburg Pasteur Institute, St. Petersburg, Russia

The goal of this study was to show the universal role of ethical conception in the realization WHO global initiative on the eradication of infectious diseases: polio, measles, rubella and congenital rubella. The analysis of the WHO Strategic Plan activities was done in the light of UNESCO Declaration "On Bioethics and Human Rights", 2005. During implementation of WHO Strategic Plan for eradication measles, rubella and congenital rubella at the national, regional and global levels was happened the obligatory need to twice postpone the deadline from 2010 to 2015 and from 2015 to 2020 . The reason of this event was connected with the lack of solidarity during preventive measures and clearly demonstrates the importance for joint actions and control over the epidemic process. The acceptation and following of these measures could help to achieve the effect, which corresponds to the ethical principals from article 13 "Solidarity and Cooperation" and article 24 "International Cooperation" of UNESCO Declaration. The diversity of situations and conditions in the implementation of the WHO program in different regions of the world, or in relation to the different cultural, social, religious, economic and psychological status of contingents requires adherence to the principles laid down in articles 8, 9, 12: "Recognition of human vulnerability and respect for personal integrity", "Equality, justice and equality" and "Respect for cultural diversity and pluralism". The whole system of administration and management of the WHO activity at each individual level of implementation should be based on the ethical principles decelerated in articles 3, 5, 14-17: "Human dignity and human rights", "Independence and individual responsibility", "Social responsibility and health", "Sharing benefits", "Protecting future generations" and "Protecting the environment, the biosphere and biodiversity." In general all elements of the implementation the global goals both in the field improving vaccine and vaccination pro- 